\title{
A Stimulus Generalization of Double Integral Inequalities by the way of Fractional Integrals
}

\author{
Muhammad Muddassar $^{{ }^{*}}$, Muhammad Iqbal ${ }^{2}$, Tahira Jabeen ${ }^{3}$ and Ghulam Haider ${ }^{1}$ \\ ${ }^{1}$ Department of Basic Sciences, University of Engineering and Technology, Taxila, Pakistan \\ ${ }^{2}$ Department of Mathematics, Govt. Graduate College, Jaranwala, Pakistan \\ ${ }^{3}$ Department of Mathematics and Statistics, International Islamic University, Islamabad, Pakistan \\ *Corresponding author
}

\begin{abstract}
This study presents some of the latest results annexed to Hermite Hadamard inequality by utilizing double integrals by the way of RiemannLiouville fractional integrals. Another aim of this article is to generalize some of the recent developments on Hermite Hadamard's type inequalities.
\end{abstract}

Keywords: Hermite- Hadamard inequality; convex functions; H older inequality; Riemann-Liouville fractional integrals.

2010 Mathematics Subject Classification: 26D10; 26A33; 26A51

\section{Introduction}

The usefulness of inequalities involving convex functions is realized from the very beginning and is now widely acknowledged as one of the prime driving forces behind the development of several modern branches of mathematics and has been given considerable attention. One of the most famous inequalities for convex functions is Hermite-Hadamard inequality, stated as [8]:

$f\left(\frac{a+b}{2}\right) \leq \frac{1}{b-a} \int_{a}^{b} f(x) d x \leq \frac{f(a)+f(b)}{2}$,

Both inequalities hold in the reversed direction for $\mathrm{f}$ to be concave.

It is well known that the Hermite-Hadamard inequality plays an important role in nonlinear analysis. In the recent years, this classical inequality has been improved and generalized in a number of ways and a large number of research papers have been written on this inequality, [8, 10, 11, 12, 13].

In recent paper, [14] Sarikaya et. al. proved a variant of Hermite-Hadamard's inequalities in fractional integral forms as follows:

Theorem 1.1. Let $f:[a, b] \rightarrow \mathbb{R}$ be a positive function with $0 \leq a<b$ and $f \in L[a, b]$. If $f$ is convex function on $[a, b]$, then the following inequalities for fractional integrals hold:

$f\left(\frac{a+b}{2}\right) \leq \frac{\Gamma(\alpha+1)}{2(b-a)^{\alpha}}\left[J_{a^{+}}^{\alpha} f(b)+J_{b^{-}}^{\alpha} f(a)\right] \leq \frac{f(a)+f(b)}{2}$

Remark 1.2. For $\alpha=1$, inequality (1.2) reduces to inequality (1.1).

In the following, we will give some necessary definitions and mathematical preliminaries of fractional calculus theory which are used further in this paper. 
Definition 1.3. Let $f \in L[a, b]$, the Reimann-Liouville integrals $J_{a^{+}}^{\alpha}$ and $J_{b^{-}}^{\alpha}$ of order $\alpha>0$ with $a \geq 0$ are defined by

$J_{a^{+}}^{\alpha}=\frac{1}{\Gamma(\alpha)} \int_{a}^{x}(x-t)^{\alpha-1} f(t) d t, \quad x>\alpha$

and

$J_{b^{-}}^{\alpha}=\frac{1}{\Gamma(\alpha)} \int_{x}^{b}(t-x)^{\alpha-1} f(t) d t, \quad x<\alpha$

respectively. Here, $\Gamma(\alpha)=\int_{0}^{\infty} e^{-t} u^{\alpha-1} d u$ is the Gamma function and $J_{a^{+}}^{0} f(x)=J_{b^{-}}^{0} f(x)=f(x)$.

In the case of $\alpha=1$, the fractional integral reduces to the classical integral. Properties concerning this operator can be found in [9].

In this article, we establish some new estimates of left and right Hermite-Hadamard inequality in the form of fractional integrals by the way of double integrals for functions whose absolute values of first derivatives are convex and concave.

\section{Main Results}

In this section, first we will establish the identities with name as lemma 2.1 and lemma 2.6 and further utilizing these two lemmas we laid down some results which estimates the left and right side of Hermite-Hadamard inequality.

Lemma 2.1. Suppose $f: \mathbb{I}^{0} \subseteq \mathbb{R} \rightarrow \mathbb{R}$ be differentiable mapping over $\mathbb{I}^{0}$ (interior of $a, b \in \mathbb{I}$ ) for $a<b$. If $\left|f^{\prime}\right|^{q}$ be a convex function and $\alpha \in(0,1)$, then it gives

$$
\begin{aligned}
& \frac{f(a)+f(b)}{2}-\frac{\gamma(\alpha+1)}{2(b-a)^{\alpha}}\left[J_{a+}^{\alpha} f(b)+J_{b-}^{\alpha} f(a)\right] \\
& \leq \frac{b-a}{2} \int_{0}^{1} \int_{0}^{1}\left[f^{\prime}(t a+(1-t) b)+f^{\prime}(s b+(1-s) a)\right]\left(s^{\alpha}-t^{\alpha}\right) d t d s
\end{aligned}
$$

Proof. Let

$$
\begin{aligned}
& I_{1}=\int_{0}^{1} \int_{0}^{1}\left(s^{\alpha}-t^{\alpha}\right) f^{\prime}(t a+(1-t) b) d t d s \\
& =\frac{\alpha}{(\alpha-1)(b-a)} f(a)+\frac{1}{(\alpha+1)(b-a)} f(b)-\frac{\alpha}{b-a} \int_{0}^{1} t^{\alpha-1} f(t a+(1-t) b) d t
\end{aligned}
$$

Similarly

$$
\begin{aligned}
& I_{2}=\int_{0}^{1} \int_{0}^{1}\left(s^{\alpha}-t^{\alpha}\right) f^{\prime}(s b+(1-s) a) d t d s \\
= & \frac{\alpha}{(\alpha+1)(b-a)} f(b)+\frac{1}{(\alpha+1)(b-a)} f(a)-\frac{\alpha}{(b-a)^{\alpha}} \int_{0}^{1} s^{\alpha-1} f(s b+(1-s) a) d s
\end{aligned}
$$

By adding (2.2) and (2.3) we have.

$$
\frac{(b-a)}{2}\left(I_{1}+I_{2}\right)=\frac{f(a)+f(b)}{2}-\frac{\gamma(\alpha+1)}{2(b-a)^{\alpha}}\left[J_{a+}^{\alpha} f(b)+J_{b-}^{\alpha} f(a)\right]
$$

Which completes the proof.

Theorem 2.2. Suppose $f: \mathbb{I}^{0} \subseteq \mathbb{R} \rightarrow \mathbb{R}$ be differentiable mapping over $\mathbb{I}^{0}$ (interior of $a, b \in \mathbb{I}$ ) for $a<b$. If $\left|f^{\prime}\right|^{q}$ be a convex function and $\alpha \in(0,1)$, then it gives.

$$
\left|\frac{f(a)+f(b)}{2}-\frac{\gamma(\alpha+1)}{2(b-a)^{\alpha}}\left[J_{a+}^{\alpha} f(b)+J_{b-}^{\alpha} f(a)\right]\right| \leq \frac{\alpha(b-a)}{(\alpha+1)(\alpha+2)}\left[\left|f^{\prime}(a)\right|+\left|f^{\prime}(b)\right|\right]
$$


Proof. From lemma 2.1

$$
\begin{aligned}
\mid \frac{f(a)+f(b)}{2}- & \frac{\gamma(\alpha+1)}{2(b-a)^{\alpha}}\left[J_{a+}^{\alpha} f(b)+J_{b-}^{\alpha} f(a)\right] \mid \\
& \leq \frac{b-a}{2} \int_{0}^{1} \int_{0}^{1}\left|\left[f^{\prime}(t a+(1-t) b)+f^{\prime}(s b+(1-s) a)\right]\left(s^{\alpha}-t^{\alpha}\right)\right| d t d s
\end{aligned}
$$

By applying convexity,

$$
\begin{aligned}
\mid \frac{f(a)+f(b)}{2}- & \frac{\gamma(\alpha+1)}{2(b-a)^{\alpha}}\left[J_{a+}^{\alpha} f(b)+J_{b-}^{\alpha} f(a)\right] \mid \\
& \leq \frac{b-a}{2} \int_{0}^{1} \int_{0}^{1}\left|\left(s^{\alpha}-t^{\alpha}\right)\right|\left(t\left|f^{\prime}(a)\right|+(1-t)\left|f^{\prime}(b)\right|\right) d t d s \\
& +\frac{b-a}{2} \int_{0}^{1} \int_{0}^{1}\left|\left(s^{\alpha}-t^{\alpha}\right)\right|\left(s\left|f^{\prime}(b)\right|+(1-s)\left|f^{\prime}(a)\right|\right) d t d s
\end{aligned}
$$

Here,

$$
\int_{0}^{1} \int_{0}^{1} t\left|\left(s^{\alpha}-t^{\alpha}\right)\right| d t d s=\frac{\alpha(3 \alpha+5)}{2(\alpha+1)(\alpha+2)(\alpha+3)}
$$

And,

$$
\int_{0}^{1} \int_{0}^{1}(1-t)\left|\left(s^{\alpha}-t^{\alpha}\right)\right| d t d s=\frac{\alpha^{2}+7 \alpha}{2(\alpha+1)(\alpha+2)(\alpha+3)}
$$

Similarly,

$$
\begin{aligned}
\int_{0}^{1} \int_{0}^{1} s\left|\left(s^{\alpha}-t^{\alpha}\right)\right| d t d s & =\frac{3 \alpha^{2}+5 \alpha}{2(\alpha+1)(\alpha+2)(\alpha+3)} \\
\int_{0}^{1} \int_{0}^{1}(1-s)\left|\left(s^{\alpha}-t^{\alpha}\right)\right| d t d s & =\frac{\alpha^{2}+7 \alpha}{2(\alpha+1)(\alpha+2)(\alpha+3)}
\end{aligned}
$$

By using above results in equation (2.6), we have.

$$
\begin{aligned}
& \left|\frac{f(a)+f(b)}{2}-\frac{\gamma(\alpha+1)}{2(b-a)^{\alpha}}\left[J_{a+}^{\alpha} f(b)+J_{b-}^{\alpha} f(a)\right]\right| \\
& \leq \frac{\alpha(b-a)}{(\alpha+1)(\alpha+2)}\left[\left|f^{\prime}(a)\right|+\left|f^{\prime}(b)\right|\right]
\end{aligned}
$$

Which completes the proof.

Theorem 2.3. Suppose $f: \mathbb{I}^{0} \subseteq \mathbb{R} \rightarrow \mathbb{R}$ be differentiable mapping over $\mathbb{I}^{0}$ (interior of $a, b \in \mathbb{I}$ ) for $a<b$. If $\left|f^{\prime}\right|^{q}$ be a convex function and $\alpha \in(0,1)$, then it gives

$$
\begin{gathered}
\left|\frac{f(a)+f(b)}{2}-\frac{\gamma(\alpha+1)}{2(b-a)^{\alpha}}\left[J_{a+}^{\alpha} f(b)+J_{b-}^{\alpha} f(a)\right]\right| \\
\left.\leq\left.\frac{2^{\frac{1}{p}}(b-a)}{(\alpha p+1)^{\frac{1}{p}}(q+1)^{\frac{1}{q}}}\left(\mid f^{\prime} a\right)\right|^{q}-\left|f^{\prime}(b)\right|^{q}\right)^{\frac{1}{q}}
\end{gathered}
$$

Proof. From lemma 2.1

$$
\begin{aligned}
& \left|\frac{f(a)+f(b)}{2}-\frac{\gamma(\alpha+1)}{2(b-a)^{\alpha}}\left[J_{a+}^{\alpha} f(b)+J_{b-}^{\alpha} f(a)\right]\right| \\
\leq & (b-a) \int_{0}^{1} \int_{0}^{1}\left|\left(s^{\alpha}-t^{\alpha}\right)\right|\left|f^{\prime}(t a+(1-t) b)\right| d t d s
\end{aligned}
$$


By applying Holder's inequality,we have.

$$
\leq(b-a)\left(\int_{0}^{1} \int_{0}^{1}\left|\left(s^{\alpha}-t^{\alpha}\right)\right|^{p} d t d s\right)^{\frac{1}{p}}\left(\int_{0}^{1} \int_{0}^{1}\left|f^{\prime}(t a+(1-t) b)\right|^{q} d t d s\right)^{\frac{1}{q}}
$$

As we know that.

$$
\left|x^{\alpha}-y^{\alpha}\right|^{p} \leq\left|x^{\alpha}+y^{\alpha}\right|^{p} \leq\left|x^{\alpha}\right|^{p}+\left|y^{\alpha}\right|^{p}
$$

So from equation (2.8), we have

$$
\int_{0}^{1} \int_{0}^{1}\left|s^{\alpha}-t^{\alpha}\right|^{p} d t d s \leq \int_{0}^{1} \int_{0}^{1}\left(\left|s^{\alpha}\right|^{p}+\left|t^{\alpha}\right|^{p}\right) d t d s \leq \frac{2}{\alpha p+1}
$$

Since $\left|f^{\prime}\right|^{q}$ is convex, we have

$$
\int_{0}^{1} \int_{0}^{1}\left|f^{\prime}(t a+(1-t) b)\right|^{q} d t d s \leq \frac{1}{q+1}\left(\left|f^{\prime}(a)\right|^{q}-\left|f^{\prime}(b)\right|^{q}\right)
$$

Now by using these values in equation (2.8), we have

$$
\left|\frac{f(a)+f(b)}{2}-\frac{\gamma(\alpha+1)}{2(b-a)^{\alpha}}\left[J_{a+}^{\alpha} f(b)+J_{b-}^{\alpha} f(a)\right]\right| \leq \frac{2^{\frac{1}{p}}(b-a)}{(\alpha p+1)^{\frac{1}{p}}(q+1)^{\frac{1}{q}}}\left(\left|f^{\prime}(a)\right|^{q}-\left|f^{\prime}(b)\right|^{q}\right)^{\frac{1}{q}}
$$

Which completes the proof.

Theorem 2.4. Suppose $f: \mathbb{I}^{0} \subseteq \mathbb{R} \rightarrow \mathbb{R}$ be differentiable mapping over $\mathbb{I}^{0}$ (interior of $a, b \in \mathbb{I}$ ) for $a<b$. If $\left|f^{\prime}\right|^{q}$ be a convex function and $\alpha \in(0,1)$, then it gives.

$$
\begin{aligned}
& \left|\frac{f(a)+f(b)}{2}-\frac{\gamma(\alpha+1)}{2(b-a)^{\alpha}}\left[J_{a+}^{\alpha} f(b)+J_{b-}^{\alpha} f(a)\right]\right| \\
& \left.\quad \leq\left.(b-a)(\ln 2)^{\frac{1}{q}}\left(\frac{2}{\alpha p+1}\right)^{\frac{1}{p}}\left(\mid f^{\prime} a\right)\right|^{q}+\left|f^{\prime}(b)\right|^{q}\right)^{\frac{1}{q}}
\end{aligned}
$$

Proof. From lemma 2.1

$$
\begin{aligned}
& \left|\frac{f(a)+f(b)}{2}-\frac{\gamma(\alpha+1)}{2(b-a)^{\alpha}}\left[J_{a+}^{\alpha} f(b)+J_{b-}^{\alpha} f(a)\right]\right| \\
& \quad \leq(b-a) \int_{0}^{1} \int_{0}^{1}\left|\left(s^{\alpha}-t^{\alpha}\right)\right|\left|f^{\prime}(t a+(1-t) b)\right| d t d s
\end{aligned}
$$

By applying Holder's inequality,

$$
\begin{aligned}
& \left|\frac{f(a)+f(b)}{2}-\frac{\gamma(\alpha+1)}{2(b-a)^{\alpha}}\left[J_{a+}^{\alpha} f(b)+J_{b-}^{\alpha} f(a)\right]\right| \\
\leq & (b-a)\left(\int_{0}^{1} \int_{0}^{1}\left|\left(s^{\alpha}-t^{\alpha}\right)\right|^{p} d t d s\right)^{\frac{1}{p}}\left(\int_{0}^{1} \int_{0}^{1}\left|f^{\prime}(t a+(1-t) b)\right|^{q} d t d s\right)^{\frac{1}{q}}
\end{aligned}
$$

By s-convexity, we have

$$
\begin{aligned}
& \int_{0}^{1} \int_{0}^{1}\left|f^{\prime}(t a+(1-t) b)\right|^{q} d t d s \leq \int_{0}^{1} \int_{0}^{1} t^{s}\left|f^{\prime}(a)\right|^{q} d t d s+\int_{0}^{1} \int_{0}^{1}(1-t)^{s}\left|f^{\prime}(b)\right|^{q} d t d s \\
& \leq \ln 2\left(\left|f^{\prime}(a)\right|^{q}+\left|f^{\prime}(b)\right|^{q}\right)
\end{aligned}
$$

And

$$
\int_{0}^{1} \int_{0}^{1}\left|s^{\alpha}-t^{\alpha}\right|^{p} d t d s \leq \frac{2}{\alpha p+1}
$$


Now by putting above values in equation (2.10), we have

$$
\begin{aligned}
& \left|\frac{f(a)+f(b)}{2}-\frac{\gamma(\alpha+1)}{2(b-a)^{\alpha}}\left[J_{a+}^{\alpha} f(b)+J_{b-}^{\alpha} f(a)\right]\right| \\
& \leq(b-a)(\ln 2)^{\frac{1}{p}}\left(\frac{2}{\alpha p+1}\right)^{\frac{1}{p}}\left(\left|f^{\prime}(a)\right|^{q}+\left|f^{\prime}(b)\right|^{q}\right)^{\frac{1}{q}}
\end{aligned}
$$

Which completes the proof.

Theorem 2.5. Suppose $f: \mathbb{I}^{0} \subseteq \mathbb{R} \rightarrow \mathbb{R}$ be differentiable mapping over $\mathbb{I}^{0}$ (interior of $a, b \in \mathbb{I}$ ) for $a<b$. If $\left|f^{\prime}\right|^{q}$ be a convex function and $\alpha \in(0,1)$, then it gives.

$$
\begin{aligned}
& \left|\frac{f(a)+f(b)}{2}-\frac{\gamma(\alpha+1)}{2(b-a)^{\alpha}}\left[J_{a+}^{\alpha} f(b)+J_{b-}^{\alpha} f(a)\right]\right| \\
& \leq(b-a)\left(\frac{1}{2 \ln 2}\right)^{\frac{1}{q}}\left(\frac{2}{\alpha p+1}\right)^{\frac{1}{p}}\left|f^{\prime}\left(\frac{a+b}{2}\right)\right|
\end{aligned}
$$

Proof. From lemma 2.1

$$
\begin{aligned}
& \left|\frac{f(a)+f(b)}{2}-\frac{\gamma(\alpha+1)}{2(b-a)^{\alpha}}\left[J_{a+}^{\alpha} f(b)+J_{b-}^{\alpha} f(a)\right]\right| \\
& \leq(b-a) \int_{0}^{1} \int_{0}^{1}\left|\left(s^{\alpha}-t^{\alpha}\right)\right|\left|f^{\prime}(t a+(1-t) b)\right| d t d s
\end{aligned}
$$

By applying Holder's inequality,we have.

$$
\begin{aligned}
& \left|\frac{f(a)+f(b)}{2}-\frac{\gamma(\alpha+1)}{2(b-a)^{\alpha}}\left[J_{a+}^{\alpha} f(b)+J_{b-}^{\alpha} f(a)\right]\right| \\
& \leq(b-a)\left(\int_{0}^{1} \int_{0}^{1}\left|\left(s^{\alpha}-t^{\alpha}\right)\right|^{p} d t d s\right)^{\frac{1}{p}}\left(\int_{0}^{1} \int_{0}^{1}\left|f^{\prime}(t a+(1-t) b)\right|^{q} d t d s\right)^{\frac{1}{q}}
\end{aligned}
$$

By s-concavity, we have

$$
\begin{gathered}
\int_{0}^{1} \int_{0}^{1}\left|f^{\prime}(t a+(1-t) b)\right|^{q} d t d s \leq\left|f^{\prime}\left(\frac{a+b}{2}\right)\right|^{q} \int_{0}^{1} \int_{0}^{1} 2^{s-1} d t d s \\
\leq\left|f^{\prime}\left(\frac{a+b}{2}\right)\right|^{q}\left(\frac{1}{2 \ln 2}\right)
\end{gathered}
$$

And

$$
\int_{0}^{1} \int_{0}^{1}\left|s^{\alpha}-t^{\alpha}\right|^{p} d t d s \leq \frac{2}{\alpha p+1}
$$

By putting above values in equation (2.12), we have.

$$
\begin{aligned}
& \left|\frac{f(a)+f(b)}{2}-\frac{\gamma(\alpha+1)}{2(b-a)^{\alpha}}\left[J_{a+}^{\alpha} f(b)+J_{b-}^{\alpha} f(a)\right]\right| \\
& \leq(b-a)\left(\frac{1}{2 \ln 2}\right)^{\frac{1}{q}}\left(\frac{2}{\alpha p+1}\right)^{\frac{1}{p}}\left|f^{\prime}\left(\frac{a+b}{2}\right)\right|
\end{aligned}
$$

Which completes the proof.

Lemma 2.6. Suppose $f: \mathbb{I}^{0} \subseteq \mathbb{R} \rightarrow \mathbb{R}$ be differentiable mapping over $\mathbb{I}^{0}$ (interior of $a, b \in \mathbb{I}$ ) for $a<b$. If $\left|f^{\prime}\right|^{q}$ be a convex function and $\alpha \in(0,1)$, then it gives.

$f\left(\frac{a+b}{2}\right)-\frac{\gamma(\alpha+1)}{2(b-a)^{\alpha}}\left[J_{a+}^{\alpha} f(b)+J_{b-}^{\alpha} f(a)\right]=\frac{b-a}{2^{\alpha+2}} \sum_{k=1}^{4} I_{k}$ 
where,

$$
\begin{gathered}
I_{1}=\int_{0}^{1} \int_{0}^{1}\left(t^{\alpha}-s^{\alpha}\right) f^{\prime}\left(t \frac{a+b}{2}+(1-t) a\right) d t d s \\
I_{2}=\int_{0}^{1} \int_{0}^{1}\left(t^{\alpha}-s^{\alpha}\right) f^{\prime}\left(s \frac{a+b}{2}+(1-s) a\right) d t d s \\
I_{3}=\int_{0}^{1} \int_{0}^{1}\left[2^{\alpha}+s^{\alpha}-(1+t)^{\alpha}\right] f^{\prime}\left(t a+(1-t) \frac{a+b}{2}\right) d t d s \\
I_{4}=\int_{0}^{1} \int_{0}^{1}\left[(1+s)^{\alpha}-2^{\alpha}-t^{\alpha}\right] f^{\prime}\left(s b+(1-s) \frac{a+b}{2}\right) d t d s
\end{gathered}
$$

Proof.

$$
\begin{aligned}
& I_{1}=\int_{0}^{1} \int_{0}^{1}\left(t^{\alpha}-s^{\alpha}\right) f^{\prime}\left(t \frac{a+b}{2}+(1-t) a\right) d t d s \\
& =\frac{2 \alpha}{(b-a)(\alpha+1)} f\left(\frac{a+b}{2}\right)+\frac{2}{(b-a)(\alpha+1)} f(a) \\
& -\frac{2 \alpha}{b-a} \int_{a}^{\frac{a+b}{2}} 2^{\alpha-1} \frac{(U-a)^{\alpha-1}}{(b-a)^{\alpha-1}} f(u) \cdot 2 \frac{d u}{b-a} \\
& I_{2}=\int_{0}^{1} \int_{0}^{1}\left(s^{\alpha}-t^{\alpha}\right) f^{\prime}\left(t \frac{a+b}{2}+(1-t) b\right) d t d s \\
& =\frac{2 \alpha}{(b-a)(\alpha+1)} f\left(\frac{a+b}{2}\right)+\frac{2}{(b-a)(\alpha+1)} f(b) \\
& -\frac{2 \alpha}{b-a} \int_{\frac{a+b}{2}}^{b} 2^{\alpha-1}\left(\frac{b-U}{b-a}\right)^{\alpha-1} f(u) \cdot 2 \frac{d u}{b-a} \\
& I_{3}=\int_{0}^{1} \int_{0}^{1}\left[2^{\alpha}+s^{\alpha}-(1+t)^{\alpha}\right] f^{\prime}\left(t a+(1-t) \frac{a+b}{2}\right) d t d s \\
& =\frac{-2}{(b-a)(\alpha+1)} f(a)+\frac{2}{b-a}\left(2^{\alpha}-\frac{\alpha}{\alpha+1}\right) f\left(\frac{a+b}{2}\right) \\
& -\frac{2 \alpha}{b-a} \int_{a}^{\frac{a+b}{2}}\left(\frac{b-u}{b-a}\right)^{\alpha-1} f(u) \frac{2 d u}{b-a} \\
& I_{4}=\int_{0}^{1} \int_{0}^{1}\left[(1+t)^{\alpha}-2^{\alpha}-s^{\alpha}\right] f^{\prime}\left(t b+(1-t) \frac{a+b}{2}\right) d t d s \\
& =\frac{-2}{(b-a)(\alpha+1)} f(b)+\frac{2}{b-a}\left(2^{\alpha}-\frac{\alpha}{\alpha+1}\right) f\left(\frac{a+b}{2}\right) \\
& -\frac{2 \alpha}{b-a} \int_{\frac{a+b}{2}}^{b} 2^{\alpha-1}\left(\frac{U-a}{b-a}\right)^{\alpha-1} f(u) \frac{2 d u}{b-a}
\end{aligned}
$$

If we accumulate above results then we have.

$$
\frac{b-a}{2^{\alpha+2}} \sum_{i=1}^{i=4} I_{i}=f\left(\frac{a+b}{2}\right)-\frac{\gamma(\alpha+1)}{(2 b-a)^{\alpha}}\left[J_{a+}^{\alpha} f(b)+J_{b-}^{\alpha} f(a)\right]
$$

Which completes the proof.

Theorem 2.7. Suppose $f: \mathbb{I}^{0} \subseteq \mathbb{R} \rightarrow \mathbb{R}$ be differentiable mapping over $\mathbb{I}^{0}$ (interior of $a, b \in \mathbb{I}$ ) for $a<b$. 
If $\left|f^{\prime}\right|^{q}$ be a convex function and $\alpha \in(0,1)$, then it gives

$$
\begin{aligned}
& \left|f\left(\frac{a+b}{2}\right)-\frac{\gamma(\alpha+1)}{2(b-a)^{\alpha}}\left[J_{a+}^{\alpha} f(b)+J_{b-}^{\alpha} f(a)\right]\right| \\
& =\frac{b-a}{2^{\alpha+2}}\left[\frac{6 \alpha^{2}+12 \alpha-4.2^{\alpha}(\alpha+3)}{2(\alpha+1)(\alpha+2)(\alpha+3)}\right]\left|f^{\prime}\left(\frac{a+b}{2}\right)\right| \\
& +\frac{b-a}{2^{\alpha+2}}\left[\frac{2 \alpha^{2}+10 \alpha+2^{\alpha}(\alpha+3)\left(\alpha^{2}-\alpha+2\right)}{2(\alpha+1)(\alpha+2)(\alpha+3)}\right]\left|f^{\prime}(a)\right| \\
& \quad+\frac{b-a}{2^{\alpha+2}}\left[\frac{6 \alpha^{2}+12 \alpha-4.2^{\alpha}(\alpha+3)}{2(\alpha+1)(\alpha+2)(\alpha+3)}\right]\left|f^{\prime}(b)\right|
\end{aligned}
$$

Proof. From lemma 2.6

$\left|f\left(\frac{a+b}{2}\right)-\frac{\gamma(\alpha+1)}{2(b-a)^{\alpha}}\left[J_{a+}^{\alpha} f(b)+J_{b-}^{\alpha} f(a)\right]\right|=\frac{b-a}{2^{\alpha+2}} \sum_{k=1}^{4}\left|I_{k}\right|$

where

$$
\begin{aligned}
\left|I_{1}\right|= & \left|\int_{0}^{1} \int_{0}^{1}\left(t^{\alpha}-s^{\alpha}\right) f^{\prime}\left(t \frac{a+b}{2}+(1-t) a\right) d t d s\right| \\
& \leq \int_{0}^{1} \int_{0}^{1}\left|t^{\alpha}-s^{\alpha}\right|\left|f^{\prime}\left(t \frac{a+b}{2}+(1-t) a\right)\right| d t d s
\end{aligned}
$$

By using convexity of $\left|f^{\prime}\right|$, we have

$$
\begin{aligned}
& \left|I_{1}\right| \leq \int_{0}^{1} \int_{0}^{1}\left|t^{\alpha}-s^{\alpha}\right|\left(t\left|f^{\prime}\left(\frac{a+b}{2}\right)\right|+(1-t)\left|f^{\prime}(a)\right|\right) d t d s \\
& \leq \int_{0}^{1} \int_{0}^{1} t\left|t^{\alpha}-s^{\alpha}\right|\left|f^{\prime}\left(\frac{a+b}{2}\right)\right| d t d s+\int_{0}^{1} \int_{0}^{1}(1-t)\left|t^{\alpha}-s^{\alpha}\right|\left|f^{\prime}(a)\right| d t d s
\end{aligned}
$$

But

$\int_{0}^{1} \int_{0}^{1} t\left|t^{\alpha}-s^{\alpha}\right| d t d s=\frac{3 \alpha^{2}+5 \alpha}{2(\alpha+1)(\alpha+2)(\alpha+3)}$

And

$\int_{0}^{1} \int_{0}^{1}(1-t)\left|t^{\alpha}-s^{\alpha}\right| d t d s=\frac{\alpha^{2}+7 \alpha}{2(\alpha+1)(\alpha+2)(\alpha+3)}$

By putting values in equation (2.16), we have

$$
\left|I_{1}\right| \leq \frac{3 \alpha^{2}+5 \alpha}{2(\alpha+1)(\alpha+2)(\alpha+3)}\left|f^{\prime}\left(\frac{a+b}{2}\right)\right|+\frac{\alpha^{2}+7 \alpha}{2(\alpha+1)(\alpha+2)(\alpha+3)}\left|f^{\prime}(a)\right|
$$

Now similarly.

$$
\begin{aligned}
\left|I_{2}\right| & =\left|\int_{0}^{1} \int_{0}^{1}\left(t^{\alpha}-s^{\alpha}\right) f^{\prime}\left(s \frac{a+b}{2}+(1-s) b\right) d t d s\right| \\
& \leq \int_{0}^{1} \int_{0}^{1}\left|t^{\alpha}-s^{\alpha}\right|\left|f^{\prime}\left(s \frac{a+b}{2}+(1-s) b\right)\right| d t d s
\end{aligned}
$$

By using convexity of $\left|f^{\prime}\right|$, and after simplification we have.

$$
\left|I_{2}\right| \leq \frac{3 \alpha^{2}+5 \alpha}{2(\alpha+1)(\alpha+2)(\alpha+3)}\left|f^{\prime}\left(\frac{a+b}{2}\right)\right|+\frac{\alpha^{2}+7 \alpha}{2(\alpha+1)(\alpha+2)(\alpha+3)}\left|f^{\prime}(b)\right|
$$


Now similarly for the next.

$$
\begin{aligned}
& \left|I_{3}\right|=\left|\int_{0}^{1} \int_{0}^{1}\left[2^{\alpha}+s^{\alpha}-(1+t)^{\alpha}\right] f^{\prime}\left(t a+(1-t) \frac{a+b}{2}\right) d t d s\right| \\
& \leq \int_{0}^{1} \int_{0}^{1}\left|2^{\alpha}+s^{\alpha}-(1+t)^{\alpha}\right|\left|f^{\prime}\left(t a+(1-t) \frac{a+b}{2}\right)\right| d t d s
\end{aligned}
$$

By convexity of $\left|f^{\prime}\right|$, and after simplification we have.

$$
\left|I_{3}\right| \leq \frac{2^{\alpha}\left(\alpha^{2}-\alpha+2\right)+\alpha}{2(\alpha+1)(\alpha+2)}\left|f^{\prime}(a)\right|+\frac{2^{\alpha}\left(\alpha^{2}+3 \alpha-6\right)+3 \alpha+8}{2(\alpha+1)(\alpha+2)}\left|f^{\prime}\left(\frac{a+b}{2}\right)\right|
$$

Now again for the next.

$$
\begin{aligned}
\left|I_{4}\right| & =\left|\int_{0}^{1} \int_{0}^{1}\left[(1+s)^{\alpha}-2^{\alpha}-t^{\alpha}\right] f^{\prime}\left(s b+(1-s) \frac{a+b}{2}\right) d t d s\right| \\
& \leq \int_{0}^{1} \int_{0}^{1}\left|(1+s)^{\alpha}-2^{\alpha}-t^{\alpha}\right|\left|f^{\prime}\left(s b+(1-s) \frac{a+b}{2}\right)\right| d t d s
\end{aligned}
$$

By convexity of $\left|f^{\prime}\right|$, and after simplification we have.

$$
\left|I_{4}\right| \leq \frac{2^{\alpha}\left(-\alpha^{2}+\alpha-2\right)-\alpha}{2(\alpha+1)(\alpha+2)}\left|f^{\prime}(b)\right|+\frac{2^{\alpha}\left(-\alpha^{2}-3 \alpha+2\right)-3 \alpha-6}{2(\alpha+1)(\alpha+2)}\left|f^{\prime}\left(\frac{a+b}{2}\right)\right|
$$

Now if we accumulate all the above results and simplify them to its lowest form then we have.

$$
\begin{aligned}
& \sum_{k=1}^{4} I_{k}=\left|I_{1}\right|+\left|I_{2}\right|+\left|I_{3}\right|+\left|I_{4}\right| \\
& \quad=\left[\frac{6 \alpha^{2}+12 \alpha-4.2^{\alpha}(\alpha+3)}{2(\alpha+1)(\alpha+2)(\alpha+3)}\right]\left|f^{\prime}\left(\frac{a+b}{2}\right)\right| \\
& \quad+\left[\frac{2 \alpha^{2}+10 \alpha+2^{\alpha}(\alpha+3)\left(\alpha^{2}-\alpha+2\right)}{2(\alpha+1)(\alpha+2)(\alpha+3)}\right]\left|f^{\prime}(a)\right| \\
& \quad+\left[\frac{6 \alpha^{2}+12 \alpha-4.2^{\alpha}(\alpha+3)}{2(\alpha+1)(\alpha+2)(\alpha+3)}\right]\left|f^{\prime}(b)\right|
\end{aligned}
$$

Now equation (2.15) becomes

$$
\begin{aligned}
& \left|f\left(\frac{a+b}{2}\right)-\frac{\gamma(\alpha+1)}{2(b-a)^{\alpha}}\left[J_{a+}^{\alpha} f(b)+J_{b-}^{\alpha} f(a)\right]\right| \\
& =\frac{b-a}{2^{\alpha+2}}\left[\frac{6 \alpha^{2}+12 \alpha-4.2^{\alpha}(\alpha+3)}{2(\alpha+1)(\alpha+2)(\alpha+3)}\right]\left|f^{\prime}\left(\frac{a+b}{2}\right)\right| \\
& +\frac{b-a}{2^{\alpha+2}}\left[\frac{2 \alpha^{2}+10 \alpha+2^{\alpha}(\alpha+3)\left(\alpha^{2}-\alpha+2\right)}{2(\alpha+1)(\alpha+2)(\alpha+3)}\right]\left|f^{\prime}(a)\right| \\
& \quad+\frac{b-a}{2^{\alpha+2}}\left[\frac{6 \alpha^{2}+12 \alpha-4.2^{\alpha}(\alpha+3)}{2(\alpha+1)(\alpha+2)(\alpha+3)}\right]\left|f^{\prime}(b)\right|
\end{aligned}
$$

Which completes the proof.

\section{Concluding Remarks}

In this paper, we obtained some of the basic results annexed to Hermite Hadamard inequality. This article is devoted to sift out several refinements, generalizations, improvements, and extensions to Hermite Hadamard inequalities. Another aim of this paper is to discuss some of the recent developments on Hermite Hadamard's type inequalities through fractional integrals. 


\section{Competing Interest}

All authors declare that they have no significant competing financial, professional, or personal interests that might have influenced the performance or presentation of the work described in this manuscript.

\section{Authors Contribution}

All authors participated to formulate this full length article.

\section{Acknowledgments}

The authors would like to thank the anonymous reviewers for their comments and suggestions.

\section{References}

[1] Anastassiou, G., Hooshmandasl, M. R., Ghasemi, A., \& Moftakharzadeh, F. Montgomery identities for fractional integrals and related fractional inequalities, J. Inequal. Pure Appl. Math. 10 (4), Article 97 (2009).

[2] Belarbi, S., \& and Dahmani, Z. On some new fractional integral inequalities, J. Inequal. Pure Appl. Math. 10 (3), Article 86 (2009).

[3] Dahmani, Z. New inequalities in fractional integrals, Int. J. Nonlinear Sci. 9 (4), 493-497 (2010).

[4] Dahmani, Z. On Minkowski and Hermite-Hadamard integral inequalities via fractional integration, Ann. Funct. Anal. 1 (1), 51-58 (2010).

[5] Dahmani, Z. Tabharit, L., \& Taf, S. New generalisations of Gruss inequality using Riemann-Liouville fractional integrals, Bull. Math. Anal. Appl. 2 (3), 93-99 (2010).

[6] Dahmani, Z., Tabharit, L., \& Taf, S. Some fractional integral inequalities, Nonl. Sci. Lett. A. 1 (2), 155-160 (2010).

[7] Dragomir, S. S., Bhatti, M. I., Iqbal, M., \& Muddassar, M. Some new fractional Integral HermiteHadamard type inequalities, J. Comp. Anal. Appl. 18 (4), 655-661 (2015).

[8] Dragomir, S. S., \& Pearce, C. E. M. Selected topics on Hermite-Hadamard inequalities and applications, RGMIA Monographs, Victoria University, 2000; Online: http://www.sta.vu.edu.au/RGMIA/monographs/hermite- hadamard.html.

[9] Gorenflo, R., \& Mainardi, F. Fractional Calculus: Integral and Differential Equations of Fractional Order, Fractals and fractional calculus in continuum mechanics (Udine, 1996), CISM Courses and Lectures, 378, Springer, Vienna, 223-276 (1997).

[10] Hussain, S., Bhatti,, M. I. \& Iqbal, M., Hadamard-type inequalities for s-convex functions I, Punjab Univ. J. Math. 41, 51-60 (2009).

[11] Kirmaci, U. S., Inequalities for differentiable mappings and applications to special means of real numbers and to midpoint formula, Appl. Math. Comput. 147, 137-146 (2004).

[12] Kirmaci, U. S., \& Ozdemir, M. E., On some inequalities for differentiable mappings and applications to special means of real numbers and to midpoint formula, Appl. Math. Comput. 153 (2), 361-368 (2004).

[13] Muddassr, M., Bhatti, M. I., \& Iqbal, M., Some New s-Hermite-Hadamard type fordifferentiable functions and their applications, Proc. Pak. Acad. Sc. 49 (1), 9-17 (2012).

[14] Sarikaya, M. Z., Set, E., Yaldiz, H., \& Basak, N., Hermite-Hadamard's Inequalities for Fractional Integrals and related Fractional Inequalities, Math. Comput. Model. 5 (9-10), 2403-2407 (2013). 